Published in final edited form as:

Acta Neuropathol. 2014 March ; 127(3): 423-439. doi:10.1007/s00401-013-1238-y.

\title{
Sequential distribution of pTDP-43 pathology in behavioral variant frontotemporal dementia (bvFTD)
}

\author{
Johannes Brettschneider\# \\ Center for Neurodegenerative Disease research (CNDR), Perelman School of Medicine at the \\ University of Pennsylvania, 3rd Floor Maloney Building, 3600 Spruce Street, Philadelphia, PA \\ 19104, USA \\ Clinical Neuroanatomy Section, Department of Neurology, Center for Biomedical research, \\ University of Ulm, Helmholtzstrasse 8/1, 89081 Ulm, Germany
}

\section{Kelly Del Tredici ${ }^{\#}$}

Clinical Neuroanatomy Section, Department of Neurology, Center for Biomedical research, University of Ulm, Helmholtzstrasse 8/1, 89081 Ulm, Germany

\section{David J. Irwin \#}

Center for Neurodegenerative Disease research (CNDR), Perelman School of Medicine at the University of Pennsylvania, 3rd Floor Maloney Building, 3600 Spruce Street, Philadelphia, PA 19104, USA

Department of Neurology, Perelman School of Medicine at the University of Pennsylvania, 3 W Gates, 3400 Spruce Street, Philadelphia, PA 19104, USA

\section{Murray Grossman}

Department of Neurology, Perelman School of Medicine at the University of Pennsylvania, $3 \mathrm{~W}$ Gates, 3400 Spruce Street, Philadelphia, PA 19104, USA

\section{John L. Robinson}

Center for Neurodegenerative Disease research (CNDR), Perelman School of Medicine at the University of Pennsylvania, 3rd Floor Maloney Building, 3600 Spruce Street, Philadelphia, PA 19104, USA

\section{Jon B. Toledo}

Center for Neurodegenerative Disease research (CNDR), Perelman School of Medicine at the University of Pennsylvania, 3rd Floor Maloney Building, 3600 Spruce Street, Philadelphia, PA 19104, USA

\section{Lubin Fang}

Clinical Neuroanatomy Section, Department of Neurology, Center for Biomedical research, University of Ulm, Helmholtzstrasse 8/1, 89081 Ulm, Germany

\section{Vivianna M. Van Deerlin}

Center for Neurodegenerative Disease research (CNDR), Perelman School of Medicine at the University of Pennsylvania, 3rd Floor Maloney Building, 3600 Spruce Street, Philadelphia, PA 19104, USA

\footnotetext{
(C) Springer-Verlag Berlin Heidelberg 2014

johannes.brettschneider@uni-ulm.de.

trojanow@upenn.edu

H. Braak, J. Q. Trojanowski are Senior authors.

Electronic supplementary material The online version of this article (doi:10.1007/s00401-013-1238-y) contains supplementary material, which is available to authorized users.
} 
Department of Pathology and Laboratory Medicine, Perelman School of Medicine at the University of Pennsylvania, 3400 Spruce Street, Philadelphia, PA 19104, USA

\author{
Albert C. Ludolph \\ Department of Neurology, University of Ulm, Oberer Eselsberg 45, 89081 Ulm, Germany
}

Virginia M.-Y. Lee

Center for Neurodegenerative Disease research (CNDR), Perelman School of Medicine at the University of Pennsylvania, 3rd Floor Maloney Building, 3600 Spruce Street, Philadelphia, PA 19104, USA

Department of Pathology and Laboratory Medicine, Perelman School of Medicine at the University of Pennsylvania, 3400 Spruce Street, Philadelphia, PA 19104, USA

\author{
Heiko Braak \\ Clinical Neuroanatomy Section, Department of Neurology, Center for Biomedical research, \\ University of Ulm, Helmholtzstrasse 8/1, 89081 Ulm, Germany
}

\title{
John Q. Trojanowski
}

Center for Neurodegenerative Disease research (CNDR), Perelman School of Medicine at the University of Pennsylvania, 3rd Floor Maloney Building, 3600 Spruce Street, Philadelphia, PA 19104, USA

Department of Pathology and Laboratory Medicine, Perelman School of Medicine at the University of Pennsylvania, 3400 Spruce Street, Philadelphia, PA 19104, USA

\# These authors contributed equally to this work.

\section{Abstract}

We examined regional distribution patterns of phosphorylated 43-kDa TAr DNA-binding protein (pTDP-43) intraneuronal inclusions in frontotemporal lobar degeneration (FTLD).

Immunohistochemistry was performed on $70 \mu \mathrm{m}$ sections from FTLD-TDP autopsy cases $(n=39)$ presenting with behavioral variant frontotemporal dementia. Two main types of cortical pTDP-43 pathology emerged, characterized by either predominantly perikaryal pTDP-43 inclusions (cytoplasmic type, cFTLD) or long aggregates in dendrites (neuritic type, nFTLD). Cortical involvement in nFTLD was extensive and frequently reached occipital areas, whereas cases with cFTLD often involved bulbar somatomotor neurons and the spinal cord. We observed four patterns indicative of potentially sequential dissemination of pTDP-43: cases with the lowest burden of pathology (pattern I) were characterized by widespread pTDP-43 lesions in the orbital gyri, gyrus rectus, and amygdala. With increasing burden of pathology (pattern II) pTDP-43 lesions emerged in the middle frontal and anterior cingulate gyrus as well as in anteromedial temporal lobe areas, the superior and medial temporal gyri, striatum, red nucleus, thalamus, and precerebellar nuclei. More advanced cases showed a third pattern (III) with involvement of the motor cortex, bulbar somatomotor neurons, and the spinal cord anterior horn, whereas cases with the highest burden of pathology (pattern IV) were characterized by pTDP-43 lesions in the visual cortex. We interpret the four neuropathological patterns in bvFTD to be consistent with the hypothesis that pTDP-43 pathology can spread sequentially and may propagate along axonal pathways.

\section{Keywords}

ALS, amyotrophic lateral sclerosis; Frontotemporal lobar degeneration; FTLD, frontotemporal dementia; FTD; Neurodegeneration; Proteinopathies; TDP-43 


\section{Introduction}

Frontotemporal lobar degeneration (FTLD) spectrum neuropathology is the second most frequent cause of dementia and, in individuals under 65 years of age, its prevalence is similar to that of Alzheimer's disease (AD) [95]. Clinically, FTLD can be divided into a behavioral variant of FTD (bvFTD) [88], a progressive disorder of executive function and social conduct, and two forms of a progressive language disorder (i.e., primary progressive aphasia; PPA) [37]: progressive non-fluent aphasia (PNFA) and semantic dementia (SD) [38]. A third PPA subtype, logopenic progressive aphasia (LPA) [37], represents an atypical language presentation of AD-neuropathology in most cases, but clinicopathological correlations in FTLD clinical syndromes are not absolute [28]. In addition, considerable phenotypical overlap exists between FTD and amyotrophic lateral sclerosis (ALS), with as many as $30 \%$ of FTD patients developing clinical symptoms of motor neuron dysfunction [65] and $50 \%$ of ALS cases displaying at least some evidence of frontal-executive cognitive deficits [82]. The link between ALS and FTLD was further strengthened by the identification of a non-coding GGGGCC hexanucleotide repeat expansion in the C9ORF72 gene which is now recognized as the most common genetic abnormality in familial and sporadic FTLD/ALS [22, 92].

The discovery of 43-kDa TAr DNA-binding protein (TDP-43) as the main component in ubiquitin-positive neuronal inclusions in the largest subset of FTLD, now referred to as FTLD-TDP, as well as in the majority of cases with ALS provided the first molecular evidence to support the concept of FTLD and ALS as being different manifestations of the same disorder, i.e., TDP-43 proteinopathy [76]. This was reinforced shortly thereafter by the discovery of mutations in the TARDBP gene encoding TDP-43 that cause FTLD-TDP and/or ALS (reviewed in [63]). The progressive accumulation of such inclusions formed by disease-specific protein aggregates is typical of most neurodegenerative diseases, including $\mathrm{AD}$ and Parkinson's disease (PD). Importantly, many of these disorders reveal a characteristic distribution pattern of their underlying pathology across specific brain regions with disease progression. For example, in $\mathrm{AD}$, tau aggregates advance from the transentorhinal cortex through the hippocampal formation into broad areas of the neocortex $[9,10]$, whereas in PD a-synuclein aggregation appears to begin mainly in the olfactory bulb and/or dorsal motor nucleus of the vagal nerve and then spread caudo-rostrally with disease progression into the midbrain, mesocortical, and neocortical regions [12, 23]. The cellular mechanisms underlying this stereotypical progression of a-synuclein pathology are incompletely understood, but increasing evidence indicates that misfolded protein aggregates can spread by a self-perpetuating process that leads to amplification and neuronto-neuron transmission of these pathologies [47, 84]. robust evidence of propagation of $A \beta$, tau, a-synuclein, and polyQ proteins has been reported [19, 60, 67, 73, 104], and studies demonstrating the seeding of TDP-43 proteinopathies also are beginning to appear [29, 78].

In a previous investigation that systematically analyzed neuropathological evidence of the propagation of phosphorylated TDP-43 (pTDP-43) in ALS, we reported that pTDP-43 lesions appear to spread from the motor neurons in brainstem motor nuclei and spinal cord motor neurons and from the agranular frontal neocortex in a frontal direction before consecutively developing in parietal and temporal neocortical areas as well as subcortical structures innervated by these cortical regions [14]. This progressive regional dissemination pattern of pTDP-43 lesions in ALS suggests that pTDP-43 aggregates may spread within the CNS via axonal transport $[11,14]$.

Previous groups have not only examined the pathology of FTD $[101,35,70]$ but also proposed progressive FTLD stages based on progression of clinical deficits [72] and progressively severe macroscopic regional brain atrophy that correlates with astrogliosis, 
neuronal loss, microvacuolation, disease duration, and severity of dementia $[17,52,53]$. To date, however, there are no studies pertaining to how the regional distribution and/or regional spread of pTDP-43 protein deposition in FTLD-TDP may or may not resemble that seen in ALS. Experimental studies suggest seeded transmission of TDP-43 pathology [2975]. Because we found preliminary evidence for a sequential dissemination of TDP-43 pathology in ALS [14] and inasmuch as ALS and FTLD are different clinical manifestations of a pathological continuum associated with TDP-43 proteinopathy [76], it seems likely that FTLD-TDP cases may also display a sequential propagation of pTDP-43 lesions. Here, we present evidence for the possible propagation of pTDP-43 in FTLD-TDP presenting as bvFTD, which could provide insight into the neuropathological determinants of selective neuronal vulnerability in FTLDTDP while, at the same time, providing essential groundwork for future strategies aimed at preventing the spread of pathology in TDP-43 proteinopathies.

\section{Materials and methods}

\section{Autopsy cohort}

Out of 60 cases with a confirmed neuropathological diagnosis of FTLD-TDP [18,69] that underwent autopsy at the University of Pennsylvania's Center for Neurodegenerative Disease research (CNDr) between 1993 and 2012 [102], we included a total of 39 (Table 1) with a clinical diagnosis of bvFTD $[1,37,88]$. Excluded were patients with neuropathologically confirmed Pick's disease (PiD) [71].

Clinical data were ascertained from an integrated clinical and autopsy database, as described previously [109], and by retrospective chart review of clinical visits within the University of Pennsylvania Health System (Table 2). Data on the neuropsychological testing included the letter-guided verbal fluency test (FAS) [59], Boston Naming Test (BNT) [49], and the MiniMental State Examination (MMSe) [27] (Table 1). Informed written consent was available according to the Declaration of Helsinki for all autopsy cases from next of kin in accordance with institutional review board guidelines of the University of Pennsylvania.

\section{Tissue preparation, staining, and immunohistochemistry}

Pathology was examined in the following regions of the central nervous system (CNS): middle frontal gyrus, orbital gyri, gyrus rectus, agranular motor cortex (Brodmann areas 4 and 6), sensory cortex, superior or middle temporal gyrus, visual cortex (Brodmann areas 17 and 18), anterior cingulate gyrus, hypothalamus, amygdala, hippocampal formation, striatum and pallidum, thalamus, midbrain (including the substantia nigra and red nucleus), pontine tegmentum at the level of the locus coeruleus, lower pons (including the motor nucleus of cranial nerve VII), medulla oblongata at level of the hypoglossal nucleus (XII), cerebellum (including both a portion of the cerebellar cortex and dentate nucleus), and cervical spinal cord (Table 2). For nine cases, olfactory bulbs were also available.

After fixation, tissue samples were embedded in paraffin using standardized cassettes, sectioned at 6-7 $\mu \mathrm{m}$, and stained with hematoxylin and eosin (HE) and for immunohistochemistry (IHC) as described elsewhere [14, 15, 32, 33]. Briefly, IHC was performed with antibodies to hyper-phosphorylated tau (monoclonal antibody PHF1; 1:1,000, gift from Dr. Peter Davies), a-synuclein (monoclonal antibody Syn303; 1:4,000, generated in CNDR) [24], pTDP-43 (rat antibody p409/410, 1:1,000, gift from Dr. Manuela Neumann) [75], and amyloid- $\beta$ (monoclonal antibody NAB228; 1:15,000; generated in CNDr) [58]. Neurofibrillary (NF) tangle stages and CERAD neuritic plaque (NP) scores are shown in Table 2. Double-labeling IHC was also performed on selected $70 \mu \mathrm{m}$ sections using the pTDP-43 antibody above together with the Vector Sg (SK-4700, Vector) blue 
chromogen and either the SMI-311 monoclonal antibody (1:1,000, Covance, Princeton, NJ, USA), the rabbit polyclonal MAP2 antibody (1:500, Millipore) or the NFH 200 monoclonal antibody (1:300, Sigma-Aldrich, St. Louis, MO, USA) with the brown chromogen 3,30diamin-obenzidine tetrahydrochloride (DAB).

To study each of the CNS regions in all 60 cases in greater neuroanatomical detail, two additional sets of $70 \mu \mathrm{m}$ sections were prepared as described previously [26] from the same paraffin blocks used immediately above as done earlier for ALS staging [14]. This thick section technique is performed on free-floating sections. It permits recognition of specific cell types and cytoarchitectonic units (subcortical nuclei and cortical layers) owing to the visualization of large numbers of biological structures and it allows accurate assessment of pathological changes, e.g., those associated with synucleinopathies, tauopathies, and pTDP-43 proteinopathies [26]. The first set of $70 \mu \mathrm{m}$ sections was stained with aldehyde fuchsin and Darrow red for topographical orientation and evaluation of neuronal loss (lipofuscin pigment deposits, basophilic Nissl material). pTDP-43 pathology was analyzed in the second set of $70 \mu \mathrm{m}$ sections using a commercially available 409/410 rabbit polyclonal antibody (1:10,000, Cosmo Bio, Carlsbad, CA, USA) and assessed according to a semiquantitative rating scale $(0$, none/not detectable or $\mathcal{2}$ aggregates per region; + , mild; + + , moderate; $3,+++$ severe/numerous in Table 2 ). In assigning cases a semiquantitative score, the extent (topographic distribution pattern) was assigned more weight than the degree of pTDP-43 pathology.

\section{Genetic analysis}

Genomic DNA was extracted from peripheral blood or brain tissue following the manufacturer's protocols (Flexi-gene Qiagen, Valencia, CA, USA) or Quick Gene DNA whole blood kit L (Autogen, Holliston, MA, USA) for blood, and QIA symphony DNA Mini Kit (Qiagen) for brain for all available cases (58/60). DNA sequencing of the entire coding region of GRN $(n=58)$ was performed in selected cases by Beckman Coulter Genomics, as described [105, 111]. Data were analyzed with Mutation Surveyor software (Soft Genetics, State College, PA, USA). C9orf72 hexanucleotide repeat expansions (defined as $>30$ repeats) were tested $(n=57)$ with repeat-primed PCR and fragment analysis, as described [16].

\section{Statistical analysis}

Data analysis was performed using SPSS (Version 17.0 SPSS Inc., Chicago, IL, USA). The "average" (and "range") of data on patient characteristics were described using the median (and interquartile range). Wilcoxon Mann-Whitney Test was used to test differences between two clinical subgroups, and Kruskal-Wallis analysis of variance on ranks was applied in case of three or more groups, followed in the event of significance by Dunn's Method. Trend analysis was conducted using the Mantel-Haenszel Chi-square test. All correlations were studied using Spearman's rank order correlation coefficient. Bonferronicorrection for multiple testing was applied when contrasts were not driven by a specific hypothesis. For all other tests, $p$ values $<0.05$ were considered significant. All statistical tests were two-sided.

\section{Results}

Two morphological types in $70 \mu \mathrm{m}$ sections associated with Mackenzie types and also with two different regional distribution patterns of aggregated pTDP-43 in neurons

Generally, pTDP-43 pathology presented as intraneuronal dash-like, skein-like, or dot-like inclusions in the perikaryon or as dystrophic neurites of varying length in the 6-7 $\mu \mathrm{m}$ thick sections as previously reported $[33,68,76]$. In $70 \mu \mathrm{m}$ sections, cortical pathology was 
characterized either by mainly intraneuronal cytoplasmic lesions with a varying amount of short dystrophic neurites (cFTLD, $n=32$ ) or by a predominance of long dystrophic neurites (nFTLD, $n=7$ ) (Fig. 1). Cases with nFTLD of pTDP-43 pathology were linked to Mackenzie type C, whereas cFTLD cases corresponded to Mackenzie types A and B (43.8 $\%$ type A, 56.2 \% type B) [68] (Table 2). Both types showed prominent involvement of projection neurons of cortical layers II and III, but cFTLD more often showed an involvement of Betz pyramidal cells of layer $\mathrm{Vb}$ in the primary motor cortex than $\mathrm{nFTLD}$.

We performed double-labeling IHC, combining pTDP-43 antibodies with antibodies to NFH, SMI-311, and MAP2 to determine whether pTDP-43 pathology in dystrophic neurites was located in axons or in dendrites (which do not express phospho-NFH) or both. pTDP-43 aggregates were present in apical as well as basal dendrites (Fig. 2). In some cases, dendritic pTDP-43 lesions protruded from the perikaryon into dendritic processes (Fig. 2a, e, g), so that affected dendrites frequently displayed bulbous swellings or protrusions (Fig. 2b, h).

Remarkably, we observed the two major morphological types to be associated with different regional distribution patterns of pTDP-43 pathology, thereby also raising the possibility of different anatomical pathways of $\mathrm{pTDP}-43$ propagation. $\mathrm{nFTLD}$ cases were characterized by top-ographically more widespread and more severe cortical pathology than cases with a predominance of cytoplasmatic pTDP-43 lesions. For example, involvement of the visual cortex (Brodmann 17, 18) was observed in $85.7 \%$ of nFTLD cases in comparison to $18.8 \%$ of cFTLD (Table 2), and cortical pTDP-43 pathology was significantly more severe in nFTLD cases, as compared to cFTLD, in the mid-frontal gyrus $(p=0.02)$, entorhinal cortex $(p=0.04)$, hippocampal formation $(p=0.04)$, anterior cingulate gyrus $(p=0.008)$, superior/ middle temporal gyrus $(p=0.001)$, angular gyrus $(p=0.002)$, sensory cortex $(p=0.04)$, and visual cortex $(p<0.001)$.

In contrast, cFTLD cases showed heavier involvement of brainstem somatomotor nuclei compared to nFTLD cases, including the motor nucleus of the hypoglossal nerve ( $p=0.03$, Suppl. Fig. 1), inferior olivary complex $(p=0.01)$, and pontine nuclei $(p=0.02)$, as well as a tendency towards more severe pTDP-43 lesions in the red nucleus $(p=0.05)$ and $a-$ motoneurons of the cervical spinal cord $(p=0.08)$.

In line with this varying topographical distribution, all 13 FTD individuals who developed clinical signs of motor neuron disease (MND) belonged to the cFTLD group (Table 2). There was a tendency towards longer survival in nFTLD cases, but this did not achieve statistical significance $(p=0.08)$.

Neuronal pTDP-43 immunoreactive inclusions (Figs. 3, 4) also were accompanied by TDP-43 positive aggregates in oligodendrocytes (Fig. 4c). As reported previously for ALS, pTDP-43 pathology was not observed in non-myelinating oligodendrocytes located along the somata of involved cortical pyramidal cells (satellite cells) [14]. Cases with nFTLD rarely had immunoreactive oligodendrocytes, whereas the cFTLD cases nearly always displayed severe oligodendroglial involvement in both brainstem and cerebrum.

\section{Four regional distribution patterns of pathology in bvFTLD-TDP}

We analyzed the regional distribution patterns of pTDP-43 in individuals who presented as bvFTD (Table 2). These cases presented mainly as cFTLD ( $n=32,82.1 \%$, Suppl. Fig. 2), whereas nFTLD was much less frequent $(n=7,17.9 \%)$.

Pattern I (Fig. 5: yellow): cases with the lowest burden of pTDP pathology (Table 2) were characterized by the presence of pTDP-43 immunoreactive inclusions in projection neurons of basal and anterior portions of the prefrontal neocortex (orbital gyri, gyrus rectus, inferior 
frontal gyrus, Figs. 1, 5a, b, d). Within the prefrontal cortex, pTDP-43 pathology occurred most extensively in projection neurons of neocortical layers II and III and was observed to a lesser extent in small projection neurons of layers V, and VI. pTDP-43 inclusions were also observed in the basolateral subnucleus of the amygdala (Fig. 5e). Neuronal pTDP-43 immunoreactive inclusions were accompanied by TDP-43 positive aggregates in oligodendrocytes.

Pattern II (Fig. 5: light orange): with increasing burden of pathology, pTDP-43 immunoreactive lesions were visible, in addition to the regions described above, in prefrontal neocortical areas located further caudal to the frontal pole, including the middle frontal gyrus, insular cortex, and anterior cingulate gyrus, and involving the same cortical layers as described above. Furthermore, pTDP-43 pathology was seen in anteromedial areas (transentorhinal region and adjoining allocortical entorhinal region and hippocampal formation, Figs. 2, 5f), the superior and middle temporal gyri, striatum, parvocellular portion of the red nucleus of the midbrain, in medial and lateral portions of the thalamus (Figs. 4a, 5e), and in precerebellar nuclei of the pons and medulla oblongata (Fig. 5c). Mild pTDP-43 pathology was observed in the anterior olfactory nucleus and olfactory tract of 9/9 cases (Fig. 3a) where the bulb was available.

Within the hippocampal formation, it was particularly the granular cells of the dentate fascia that showed extensive pTDP-43 pathology (Fig. 3b-d), accompanied by inclusions in pyramidal neurons of Ammon's horn (CA), including sectors CA1-CA2 (Figs. 3e, 5f). In the entorhinal cortex, pTDP-43 inclusions were mainly observed in layers pre- $\alpha$, pre- $\beta$, and, to a lesser extent, in pri-a (Fig. 3f).

Striatal lesions were seen mainly in the ventral striatum (accumbens nucleus, Fig. 3f, g) but also in the putamen within medium-sized projection neurons. The projection neurons of the claustrum also contained pTDP-43 pathology, whereas inclusions were not observed in the pallidum (Fig. 5e). Representative of the involvement of the precerebellar nuclei [13] was the inferior olivary complex. Within this complex, pTDP-43 inclusions occurred chiefly in circumscribed groups of neurons within ventral portions of the principal olivary nucleus (Fig. 4b), within the dorsal accessory olivary nucleus, and, more infrequently, in the medial accessory olive. Similarly to the inferior olivary complex, pTDP-43 aggregates in the pontine gray matter were seen in isolated groups of neurons, forming an insular pattern. Inasmuch as the neurons in the parvocellular red nucleus also contained pTDP-43 pathology and are known to project to the inferior olivary complex, we assessed the pathology there within the context of the precerebellar nuclei.

Pattern III (Fig. 5: dark orange): cases with additional pathology beyond what was described above were characterized by the presence of pTDP-43 immunoreactive inclusions in the agranular motor neocortex (Brodmann fields 4 and 6), in parietal neocortical areas (sensory cortex, angular gyrus) (Fig. 5b), and in bulbar somatomotor neurons as well as the cervical spinal cord anterior horns. Within the motor cortex, pTDP-43 pathology predominated in projection neurons of layers II and III, but also in the primary motor field in Betz pyramidal cells of layer $\mathrm{Vb}$ and in projection neurons of layers $\mathrm{V}-\mathrm{VI}$. In the anterior horn of the spinal cord, pTDP-43 pathology was present in a-motoneurons of lamina 9 (Fig. 4c). Notably, oligodendrocytes of the anterior and lateral portions of the corticospinal tract were also pTDP-43-immunoreactive (Fig. 4c). In the lower brainstem, pTDP-43 pathology was present bilaterally in the hypoglossal nucleus (XII) (Figs. 4d-f, 5c)—and, occasionally, to a mild extent in intramedullary axons arising from the hypoglossal motor nucleus (Fig. 4f) - in the ambiguus nucleus of the vagal nerve (X), and in motor nuclei of cranial nerves XI, VII, and $\mathrm{V}$. In contrast, the motor neurons of cranial nerves III, IV, and VI did not display pTDP-43 aggregates (see above). Similarly, brainstem nuclei that remained free of pTDP-43 
pathology included the serotoninergic nuclei of the raphe system and noradrenergic locus coeruleus.

Pattern IV (Fig. 5: red): cases with the highest burden of pathology showed the presence of pTDP-43 lesions in the occipital neocortex (visual cortex, Brodmann 17, 18, Figs. 4g, 5a, b, d) in addition to involvement of all of the areas described above. Finally, a minority of stage 4 cases $(n 4)$ also displayed mild involvement of the dentate nucleus in the cerebellum, whereas the cerebellar cortical gray matter generally remained unaffected by pTDP-43 pathology.

\section{Genetic and clinicopathological correlations}

Across all FTLD cases, the extent of pTDP-43 pathology correlated with neuronal loss in many cortical and subcortical regions (Suppl. Table 1). The severity of the pTDP-43 pathology did not correlate with age of onset, although we found that the more severe pTDP-43 pathology in the midbrain, medulla oblongata, and cervical spinal cord was associated with a shorter duration of disease to death interval (Suppl. Table 1).

Clinical symptoms of MND were observed in 13 cases $(33.3 \%)$, and were absent in 23 cases $(59 \%)$, and data were unavailable for 3 cases $(7.7 \%)$. Cases with clinical signs of MND showed significantly more severe pTDP-43 pathology in the primary motor cortex $(p 0.001)$, pons $(p=0.001)$, hypoglossal nucleus XII $(p=0.04)$, and in the cervical spinal cord anterior horn $(p=0.04)$ compared to cases without MND clinical signs. reduced output on the letter fluency test (FAS test) was correlated with greater TDP-43 pathology in the middle frontal gyrus $(\rho=-0.41, p=0.04)$ and the angular gyrus $(\rho-0.44, p=0.03)$. No further correlation between pTDP-43 pathology and performance in neuropsychological testing was detectable.

Our cohort included 12 cases with the $C 9$ orf72 hexa-nucleotide expansion $(30.8 \%)$ and 24 cases $(61.5 \%)$ in which the expansion was absent. In 3 cases $(7.7 \%)$, data pertaining to the C9orf72 expansion was missing. For the most part, cases with the C9orf7 expansion presented with cFTLD pathology ( $n=11 / 12,91.7 \%)$, corresponding to $75 \%$ Mackenzie type $\mathrm{B}, 8.3 \%$ type $\mathrm{A}$, and $16.7 \%$ type $\mathrm{C}$ [68], and frequently showed clinical signs of MND $(50 \%)$. pTDP-43 pathology was more severe in the sensory cortex of cases with the C9orf72 expansion ( $p$ 0.04) but did not significantly differ in any of the other regions examined (data not shown). There was no significant difference between cases with and without the C9orf72 expansion regarding disease duration and onset to death interval or age at onset ( $p 0.3$ each). Furthermore, our study included 6 cases with a $G R N$ mutation. Cases with a GRN mutation did not show a significant difference as compared to cases with no mutation regarding age of onset or disease duration ( $p=0.7$ each). All cases with a GRN mutation presented as cFTLD and Mackenzie type A [68] and showed significantly less pTDP-43 pathology in the entorhinal cortex $(p=0.04)$, hippocampus $(p=0.04)$, and the CSC $(p=0.008)$ as compared to cases without GRN mutation.

\section{Discussion}

Evidence of selective degeneration of pTDP-43 inclusion-bearing neurons according to regional and sequential patterns supports the concept that pTDP-43 aggregates are closely associated with neurodegeneration [57]. Both we and others have reported that the severity of pTDP-43 pathology correlates with neuronal loss across many regions of the CNS [15, 77]. Although the precise molecular and neurobiological mechanisms of pTDP-43-mediated neurodegeneration still must be clarified, it is recognized that a loss of physiological TDP-43 function that entails neuronal dysfunction and death could be the result of toxic gains of function owing to phosphorylation, aggregation and cleavage, or losses of normal function owing to clearance of pTDP-43 from the cell nucleus [57, 63]. 
The current study provides preliminary evidence for a sequential regional dissemination of pTDP-43 pathology and, furthermore, it indicates that the mechanisms of pTDP-43 progression in FTLD presenting as bvFTD and ALS may be similar. To determine a potential progression of pTDP-43 aggregates, we applied an approach based on the assumption that intraneuronal pathological lesions probably develop consecutively at CNS sites and then increase in severity as well as extent of CNS involvement with disease progression. This rationale is in line with our recent findings on the progressive spread of pTDP-43 pathology in ALS [14] and a new study characterizing the sequential spread of TDP-43 in AD [44] as well as with previous studies aimed at analyzing the topographical distribution patterns of pathology in other neurodegenerative diseases, such as AD and PD $[10,12,80]$.

\section{Types of neocortical pTDP-43 pathology}

In $70 \mu \mathrm{m}$ sections from the cases studied here, we observed two major types of cortical pTDP-43 pathology: cFTLD was characterized by predominantly cytoplasmic neuronal inclusions (with variable presence of short dystrophic neurites), and nFTLD by long dystrophic neurites, which could be identified as dendrites using double-labeling IHC, and by less abundant cytoplasmic inclusions (Fig. 2). These subdivisions constitute a simplification of the previously described four morphological types of cortical pTDP-43 pathology based on an analysis of 7-10 $\mu \mathrm{m}$ sections [68]. In $70 \mu \mathrm{m}$ sections, cFTLD approximated Mackenzie types A and B, whereas nFTLD approximated Mackenzie type C (Table 2). The Mackenzie types of cortical pTDP-43 pathology were not readily ascertained in $70 \mu \mathrm{m}$ sections [26], where we observed that the pTDP-43 pathology was much more widely and severely distributed across all neo-cortical layers (Fig. 1). Furthermore, we think that thick slices disambiguate the two types of neuritic inclusions reported in thin sections that may have cross-cut the neurites. In addition, we observed essentially different regional distribution patterns for the two types we propose here, and these patterns, in turn, make it possible to speculate that two different pathways of pTDP-43 progression exist in FTLD.

Whereas cases with nFTLD pathology showed widespread and severe neocortical involvement from orbito-frontal areas along the entire convexity of the brain that frequently reached the occipital cortex in the most advance cases (Fig. 4g), pTDP-43 lesions were comparatively scarce in brainstem somatomotor nuclei, the precerebellar nuclei, and the anterior horn of the cervical spinal cord (Suppl. Fig. 1). This observation is consonant with prior reports that Mackenzie type $\mathrm{C}$ cases display only mild involvement of the diencephalon, brainstem, and spinal cord anterior horn [45, 46]. In contrast, cFTLD cases showed less severe neocortical involvement, whereas bulbar somatomotor neurons and the spinal cord anterior horns were more involved. While neocortical layers II and III were extensively involved in both types (Fig. 1), pTDP-43 lesions in Betz pyramidal cells of layer $\mathrm{Vb}$ in the primary motor cortex were more frequent in cFTLD. Inasmuch as these cells are the origin of major corticofugal projections in the corticospinal and corticobulbar tracts, it is plausible that they contribute to the more frequent involvement of bulbar somatomotor neurons and spinal cord a-motoneurons encountered in cFTLD.

Consequently, within the continuum of pTDP-43 proteinopathies [51, 83], cFTLD type pathology appears to be more closely related to AlS than nFTLD both with regard to neuronal pTDP-43 lesion morphology and the anatomical/regional distribution pattern of the lesions $[32,33,76]$. Notably, these differing distribution patterns were of significant clinical relevance: cFTLD cases more frequently displayed clinical signs of MND $(p<0.001)$ and showed a tendency towards a shorter survival period as compared to nFTLD cases (Table 2).

The two morphological types of neocortical pTDP-43 distribution observed here could point to the existence of different neuronal mechanisms for responding to toxic pTDP-43 protein 
assemblies [57]. Whereas some neurons (e.g., in nFTLD) can localize pTDP-43 aggregates to the dendritic compartment, this might not be possible for other neurons (e.g., in cFTLD). The cellular mechanisms underlying these different morphological distributions of pTDP-43 lesions are unknown. There is increasing recognition of possible prion-like phenomena of cell-to-cell transmission in neurodegenerative diseases [84, 85], and the C-terminal domain of TDP-43 shows structural similarities to prions [78]. given the acknowledged relevance of various strains of the pathogenic prion protein for disease heterogeneity in prion diseases $[20,30,96]$, it can be speculated that pTDP-43 may also form diverse strains of pathogenic protein that may be associated with a differential morphology of pTDP-43 inclusions as well as with different spreading patterns - and, hence, varying clinical phenotypes in FTLD. While this has not yet been described for TDP-43, at least two different strains of pathological a-synuclein have been described by guo et al. [39] and it is likely that other non-prion neurodegenerative disease proteins will be shown to exist as different strains of the same pathological protein.

\section{Spreading of pathology in FTLD-TDP presenting as bvFTD}

We analyzed the possible spreading of pTDP-43 pathology in cases that had presented with bvFTD (Table 2). given the clinical, pathological, and genetic heterogeneity of bvFTD [83, 97], the cohort size $(n=39)$ was too small to permit our proposing a neuropathological staging procedure for bvFTLD, as done previously for ALS [14]. Nonetheless, we observed the existence of four characteristic regional distribution patterns indicative of sequential pTDP-43 dissemination. Additional studies are required to determine whether the four patterns we describe here are disparate or, alternatively, when taken together, form a continuum ranging from pattern I to pattern IV. In contrast to the reports of previous authors, our findings indicate that (1) the degree of protein deposition (here, the severity of TDP-43-associated pathology) tends to be related (i.e., increase) in successive patterns [53, 54], and (2) the primary visual cortex and lower brainstem will be useful for future immunohistochemically-based staging protocols $[17,50]$. On the other hand, our data also enable us to note that some of the earliest TDP-43-associated lesions occur in the orbitofrontal cortex $[17,50]$. The data for pattern I, however, show that the amygdala is also among the earliest preferentially vulnerable sites in FTLD-TDP presenting as bvFTD (see below).

In cases with the lowest burden of pTDP-43 pathology (pattern I), widespread pTDP-43 lesions were observed in basal parts of the prefrontal neocortex (orbital gyri, gyrus rectus) (Fig. 5a, b, d). The orbitofrontal cortex is thought to play a pivotal role in executive function and emotional enhancement of memory $[56,100]$ and it is characterized by widespread connections with primary olfactory areas, high-order sensory association and other prefrontal cortical areas, with the amygdala, and with memory-related temporal cortices [3].

Our observations of early pTDP-43 pathology in the prefrontal cortex confirm earlier descriptions of severe pTDP-43 lesions in prefrontal neocortical areas of cases with FTLD $[18,33,45,68,76,98]$ and corroborate neuroimaging findings of prefrontal brain atrophy in FTD $[36,41,55,62,81,94,99,106]$.

pTDP-43 lesions were abundant in layers II and III of the prefrontal neocortex (Fig. 1), and both layers are closely linked to adjacent neocortical areas via association fibers, thereby providing a potential pathway for pTDP-43 dissemination within the neocortex $[25,66$, 108]. It has been suggested that cortico-cortical connections directed from less differentiated cortices (e.g., the phylogenetically young prefrontal cortex) towards primary areas with a higher degree of laminar differentiation originate primarily from deeper cortical layers (e.g., layers 5,6 ) and terminate in upper cortical layers (layers I, II, III) $[6,93]$. Spreading of 
pTDP-43 pathology via such connections within the pre-frontal cortex could contribute to the pronounced involvement of the upper cortical layers in FTLD.

Pattern I was also characterized by an involvement of basolateral portions of the amygdala, which is in line with previous reports of pTDP-43 inclusions and neuronal loss [33, 52] as well as with neuropathology and neuroimaging findings of amygdalar atrophy in patients with FTD [7, 52, 64, 87, 107]. Axons from the amygdala terminate in layers I-II of many prefrontal fields [86], and the prefrontal limbic areas in the posterior orbitofrontal and anterior cingulate cortex send major projections to the amygdala [34]. Therefore, close anatomical interconnectivities exist between the orbitofrontal cortex and the amygdala, and these, in turn, could also facilitate early dissemination of pTDP-43 pathology in FTLD. Inasmuch as the number of cases with a low burden of pTDP-43 pathology in this study was limited, additional efforts are needed to confirm the earliest distribution of the lesions in FTLD presenting as bvFTD.

With increasing burden of pTDP-43 pathology, the lesions occurred in additional prefrontal areas adjacent to the orbital gyri and/or gyrus rectus (e.g., the middle frontal gyrus) and also appeared in the anterior cingulate gyrus, mediotemporal cortex, and hippocampal formation (pattern II, Fig. 3). early involvement of entorhinal areas in FTLD could be facilitated by cortico-cortical projections from the orbitofrontal gyrus to the entorhinal cortex, which projects to the hippocampus [91].

Hippocampal neuronal damage is usually linked to episodic memory deficits [21, 43], and clinical observations indicate that patients with bvFTD experience episodic memory deficits, which may reach a magnitude similar to AD in some individuals [42, 83]. Indeed, neuroimaging studies show hippocampal atrophy in bvFTD patients that can be as severe as that seen in $\mathrm{AD}[74,103]$.

The anterior cingulate gyrus is part of the prefrontal limbic system [110] and receives cortico-cortical afferences from the posterior orbitofrontal cortex [3], thereby providing a possible pathway for early propagation of pTDP-43 pathology. Similar to the orbitofrontal cortex, the anterior cingulate gyrus is known to have dense connections with the basolateral nucleus of the amygdala [4].

As in previous studies, we encountered pTDP-43 lesions in medium spiny projection neurons of the striatum $[2,33]$, which were most severe in the accumbens nucleus (Fig. 3f, $\mathrm{g})$. Such striatal involvement is reflected by neuroimaging findings of striatal atrophy in cases with bvFTD [31]. Striatal pTDP-43 pathology in the putamen could be anticipated to cause extrapyramidal motor symptoms (e.g., bradykinesia or rigidity). Indeed, all of the bvFTD cases with clinically detectable signs of extrapyramidal motor deficits showed extensive pTDP-43 lesions in the putamen (Table 2). However, accumulating pTDP-43 aggregates are likely to only gradually impair neuronal function; therefore appearance of clinical symptoms could considerably trail behind the occurrence of pTDP-43 lesions [14]. The accumbens nucleus has been associated with emotional/ motivational functions of the striatum [90] and is closely connected to limbic structures (amygdala, hippocampus) as well as the prefrontal neocortex [40]. Moreover, lesions in ventral striatal regions have been associated with various forms of behavioral dysregulation, e.g., impulsivity [8]. As such, early involvement of the accumbens nucleus by pTDP-43 pathology could contribute to some of the neuropsychological deficits reported in individuals with bvFTD [79]. Cases corresponding to patterns I and II furthermore displayed TDP-43 pathology in portions of the thalamus (Fig. 4a), which is closely connected with the orbitofrontal gyrus and other prefrontal cortical areas [5]. Intriguingly, all regions that we observed to be involved in early pTDP-43 dissemination in bvFTD (patterns I and II) are characterized by close anatomical 
connections with the orbitofrontal cortex. Although the small number of early stage cases (patterns I, II, Table 2) preclude any definitive statements regarding the earliest onset of FTLD that presents as bvFTD, our data strongly point to an important role of the orbital gyri/gyrus rectus in the early dissemination of pTDP-43 pathology. In cases with the third pattern (III), involvement of the primary motor cortex, bulbar somato-motor neurons, and the cervical spinal cord anterior horn (Fig. 4) is consonant with previous neuropathological findings $[2,18,33,76]$ and with clinical reports that up to $30 \%$ of FTD patients develop MND signs $[61,65]$. While all of our bvFTD cases with clinical signs of MND displayed a pronounced involvement of the agranular neocortex, the pTDP-43 lesions in the hypoglossal nucleus and the spinal cord anterior horn were less prominent in comparison to those seen in ALS [14].

Cases with the highest burden of pTDP-43 pathology (pattern IV) were characterized by the additional presence of pTDP-43 inclusions in occipital neocortical areas (Brodmann areas 17, 18, Fig. 4g). This involvement of the occipital neocortex was more frequent than in ALS [14] and it was predominantly seen in cases with nFTLD (Suppl. Fig. 1). Visual deficits have not been described as a leading or characteristic symptom of bvFTD. However, for the most part, pTDP-43 lesions in the occipital cortex were mild, and it is plausible that this pathology might only gradually impair neuronal function before crossing a threshold that leads to neuronal death [57].

Whereas genetic subtypes of FTLD-TDP presenting as bvFTD (e.g., cases with $C 9$ orf 72 repeat expansion or $G R N$ mutation carriers) displayed some differences regarding the regional severity of pTDP-43 pathology, the overall patterns were similar and could indicate a common underlying spreading scheme. However, the number of C9orf72 expansion carriers and cases with $G R N$ mutation included here were comparatively small, underlining the need for further studies on possible differences in TDP-43 propagation among these genetic subtypes.

\section{Possible mechanisms of pTDP-43 dissemination in FTLD}

If, as implied by several groups [47, 48, 89], cell-to-cell transmission of pathological TDP-43 takes place between contiguous CNS cell types, then the pTDP-43 inclusion pathology in FTLD-TDP should occur diffusely throughout the CNS rather than in specific types of nerve cells within circumscribed regions, as reported here. Moreover, as in ALS, neurons without major cortical input, such as the dorsal nucleus of the vagal nerve or the locus coeruleus, remain intact or become only slightly involved in FTLD-TDP. The four regional distribution patterns of FTLD-TDP presenting as bvFTD appear to us to be more consistent with the neuron-to-neuron transfer of pathological TDP-43 along axonal projections, a hypothesis we proposed recently for ALS [14]. This also implies a common mechanism of pTDP-43 dissemination in TDP-43 proteinopathies. The possible phenomenon of axonal spreading in TDP-43 proteinopathies also gains support from the pattern of oligodendroglial involvement in both FTLD-TDP and ALS, with pTDP-43 lesions having been observed in oligodendrocytes in close proximity to involved axons, whereas the satellite cells of the neuronal soma remain free of pTDP-43 inclusions [14]. The directionality of axonal pTDP-43 dissemination has to be resolved by future studies that accurately model pTDP-43 proteinopathies [11].

\section{Supplementary Material}

Refer to Web version on PubMed Central for supplementary material. 


\section{Acknowledgments}

We thank the many patients who contributed to this study. We are also grateful to Kevin Raible, Terry Schuck, Sigrid Baumann, Gabriele Ehmke, Simone Feldengut, Julia Straub, and Thi Phuong Thu Brettschneider for technical support, together with David Ewert (University of Ulm) for assistance with the graphics. This study was supported by the NIH (AG033101, AG017586, AG010124, AG032953, AG039510, NS044266), the Wyncote Foundation, and the Koller Family Foundation. VM-YL is the John H. Ware, $3^{\text {rd }}$, Professor of Alzheimer's Disease research. JQT is the William Maul Measey-Truman G. Schnabel, Jr. Professor of Geriatric Medicine and Gerontology. DJI is supported by T32-Ag000255. This study was supported by the German BMBF FTLD Consortium.

\section{References}

1. Armstrong MJ, Litvan I, Lang AE, et al. Criteria for the diagnosis of corticobasal degeneration. Neurology. 2013; 80:496-503. [PubMed: 23359374]

2. Armstrong RA, Carter D, Cairns NJ. A quantitative study of the neuropathology of 32 sporadic and familial cases of frontotemporal lobar degeneration with TDP-43 proteinopathy (FTLD-TDP). Neuropathol Appl Neurobiol. 2012; 38:25-38. [PubMed: 21696412]

3. Barbas H. Specialized elements of orbitofrontal cortex in primates. Ann N Y Acad Sci. 2007; 1121:10-32. [PubMed: 17698996]

4. Barbas H, De Olmos J. Projections from the amygdala to basoventral and mediodorsal prefrontal regions in the rhesus monkey. J Comp Neurol. 1990; 300:549-571. [PubMed: 2273093]

5. Barbas H, Henion TH, Dermon CR. Diverse thalamic projections to the prefrontal cortex in the rhesus monkey. J Comp Neurol. 1991; 313:65-94. [PubMed: 1761756]

6. Barbas H, Pandya DN. Architecture and intrinsic connections of the prefrontal cortex in the rhesus monkey. J Comp Neurol. 1989; 286:353-375. [PubMed: 2768563]

7. Barnes J, Whitwell JL, Frost C, et al. Measurements of the amygdala and hippocampus in pathologically confirmed Alzheimer disease and frontotemporal lobar degeneration. Arch Neurol. 2006; 63:1434-1439. [PubMed: 17030660]

8. Basar K, Sesia T, Groenewegen H, et al. Nucleus accumbens and impulsivity. Prog Neurobiol. 2010; 92:533-557. [PubMed: 20831892]

9. Braak H, Alafuzoff I, Arzberger T, et al. Staging of Alzheimer disease-associated neurofibrillary pathology using paraffin sections and immunocytochemistry. Acta Neuropathol. 2006; 112:389404. [PubMed: 16906426]

10. Braak H, Braak E. Neuropathological stageing of Alzheimer-related changes. Acta Neuropathol. 1991; 82:239-259. [PubMed: 1759558]

11. Braak H, Brettschneider J, Ludolph A, et al. Amyotrophic lateral sclerosis: a model of corticofugal axonal spread. Nat Rev Neurol. 2013; 9:708-714. [PubMed: 24217521]

12. Braak H, Del Tredici K, Rüb U, et al. Staging of brain pathology related to sporadic Parkinson's disease. Neurobiol Aging. 2003; 24:197-211. [PubMed: 12498954]

13. Braak H, Rüb U, Del Tredici K. Involvement of precerebellar nuclei in multiple system atrophy. Neuropathol Appl Neurobiol. 2003; 29:60-76. [PubMed: 12581341]

14. Brettschneider J, Del Tredici K, Toledo JB, et al. Stages of pTDP-43 pathology in amyotrophic lateral sclerosis. Ann Neurol. 2013; 74:20-38. [PubMed: 23686809]

15. Brettschneider J, Libon DJ, Toledo JB, et al. Microglial activation and TDP-43 pathology correlate with executive dysfunction in amyotrophic lateral sclerosis. Acta Neuropathol. 2012; 123:395407. [PubMed: 22210083]

16. Brettschneider J, Van Deerlin VM, Robinson JL, et al. Pattern of ubiquilin pathology in ALS and FTLD indicates presence of C9ORF72 hexanucleotide expansion. Acta Neuropathol. 2012; 123:825-839. [PubMed: 22426854]

17. Broe M, Hodges JR, Schofield E, et al. Staging disease severity in pathologically confirmed cases of frontotemporal dementia. Neurology. 2003; 60:1005-1011. [PubMed: 12654969]

18. Cairns NJ, Bigio EH, Mackenzie IR, et al. Neuropathologic diagnostic and nosologic criteria for frontotemporal lobar degeneration: consensus of the consortium for frontotemporal lobar degeneration. Acta Neuropathol. 2007; 114:5-22. [PubMed: 17579875] 
19. Clavaguera F, Bolmont T, Crowther RA, et al. Transmission and spreading of tauopathy in transgenic mouse brain. Nat Cell Biol. 2009; 11:909-913. [PubMed: 19503072]

20. Colby DW, Prusiner SB. De novo generation of prion strains. Nat rev Microbiol. 2011; 9:771-777. [PubMed: 21947062]

21. De Leon MJ, Convit A, George AE, et al. In vivo structural studies of the hippocampus in normal aging and in incipient Alzheimer's disease. Ann N Y Acad Sci. 1996; 777:1-13. [PubMed: 8624070]

22. Dejesus-Hernandez M, Mackenzie IR, Boeve BF, et al. Expanded GGGGCC hexanucleotide repeat in noncoding region of C9ORF72 causes chromosome 9p-linked FTD and ALS. Neuron. 2011; 72:245-256. [PubMed: 21944778]

23. Dickson DW, Braak H, Duda JE, et al. Neuropathological assessment of Parkinson's disease: refining the diagnostic criteria. Lancet Neurol. 2009; 8:1150-1157. [PubMed: 19909913]

24. Duda JE, Giasson BI, Mabon ME, et al. Novel antibodies to synuclein show abundant striatal pathology in Lewy body diseases. Ann Neurol. 2002; 52:205-210. [PubMed: 12210791]

25. Fang PC, Stepniewska I, Kaas JH. Ipsilateral cortical connections of motor, premotor, frontal eye, and posterior parietal fields in a prosimian primate, Otolemur garnetti. J Comp Neurol. 2005; 490:305-333. [PubMed: 16082679]

26. Feldengut S, Del Tredici K, Braak H. Paraffin sections of 70-100mum: a novel technique and its benefits for studying the nervous system. J Neurosci Methods. 2013; 215:241-244. [PubMed: 23537935]

27. Folstein MF, Folstein Se, Mchugh Pr. "Mini-mental state". A practical method for grading the cognitive state of patients for the clinician. J Psychiatr res. 1975; 12:189-198. [PubMed: 1202204]

28. Forman MS, Farmer J, Johnson JK, et al. Frontotemporal dementia: clinicopathological correlations. Ann Neurol. 2006; 59:952-962. [PubMed: 16718704]

29. Furukawa Y, Kaneko K, Watanabe S, et al. A seeding reaction recapitulates intracellular formation of Sarkosyl-insoluble transactivation response element (TAR) DNA-binding protein-43 inclusions. J Biol Chem. 2011; 286:18664-18672. [PubMed: 21454603]

30. Gambetti P, Cali I, Notari S, et al. Molecular biology and pathology of prion strains in sporadic human prion diseases. Acta Neuropathol. 2011; 121:79-90. [PubMed: 21058033]

31. Garibotto V, Borroni B, Agosti C, et al. Subcortical and deep cortical atrophy in frontotemporal lobar degeneration. Neurobiol Aging. 2011; 32:875-884. [PubMed: 19501427]

32. Geser F, Brandmeir NJ, Kwong LK, et al. Evidence of multisystem disorder in whole-brain map of pathological TDP-43 in amyotrophic lateral sclerosis. Arch Neurol. 2008; 65:636-641. [PubMed: 18474740]

33. Geser F, Martinez-lage M, Robinson J, et al. Clinical and pathological continuum of multisystem TDP-43 proteinopathies. Arch Neurol. 2009; 66:180-189. [PubMed: 19204154]

34. Ghashghaei HT, Hilgetag CC, Barbas H. Sequence of information processing for emotions based on the anatomic dialogue between prefrontal cortex and amygdala. NeuroImage. 2007; 34:905923. [PubMed: 17126037]

35. Giannakopoulos P, Hof PR, Bouras C. Dementia lacking distinctive histopathology: clinicopathological evaluation of 32 cases. Acta Neuropathol. 1995; 89:346-355. [PubMed: 7610766]

36. Gordon E, Rohrer JD, Kim LG, et al. Measuring disease progression in frontotemporal lobar degeneration: a clinical and MRI study. Neurology. 2010; 74:666-673. [PubMed: 20177120]

37. Gorno-Tempini ML, Hillis AE, Weintraub S, et al. Classification of primary progressive aphasia and its variants. Neurology. 2011; 76:1006-1014. [PubMed: 21325651]

38. Grossman M. Primary progressive aphasia: clinicopatho-logical correlations, nature reviews. Neurology. 2010; 6:88-97. [PubMed: 20139998]

39. Guo, Jl; Covell, DJ.; Daniels, JP., et al. Distinct alpha-synuclein strains differentially promote tau inclusions in neurons. Cell. 2013; 154:103-117. [PubMed: 23827677]

40. Haber SN. The primate basal ganglia: parallel and integrative networks. J Chem Neuroanat. 2003; 26:317-330. [PubMed: 14729134] 
41. Hornberger M, Geng J, Hodges JR. Convergent grey and white matter evidence of orbitofrontal cortex changes related to disinhibition in behavioural variant frontotemporal dementia. Brain $\mathrm{J}$ Neurol. 2011; 134:2502-2512.

42. Hornberger M, Savage S, Hsieh S, et al. Orbitofrontal dysfunction discriminates behavioral variant frontotemporal dementia from Alzheimer's disease. Dement geriatr Cogn Disord. 2010; 30:547552. [PubMed: 21252550]

43. Jack CR Jr, Petersen RC, Xu YC, et al. Medial temporal atrophy on MRI in normal aging and very mild Alzheimer's disease. Neurology. 1997; 49:786-794. [PubMed: 9305341]

44. Josephs KA, Murray ME, Whitwell JL, et al. Staging TDP-43 pathology in Alzheimer's disease. Acta neuropathologica. 2013 Epub ahead of print.

45. Josephs KA, Stroh A, Dugger B, et al. Evaluation of sub-cortical pathology and clinical correlations in FTID-U sub-types. Acta Neuropathol. 2009; 118:349-358. [PubMed: 19455346]

46. Josephs KA, Whitwell JL, Murray ME, et al. Corticospinal tract degeneration associated with TDP-43 type C pathology and semantic dementia. Brain J Neurol. 2013; 136:455-470.

47. Jucker M, Walker LC. Self-propagation of pathogenic protein aggregates in neurodegenerative diseases. Nature. 2013; 501:45-51. [PubMed: 24005412]

48. Kanouchi T, Ohkubo T, Yokota T. Can regional spreading of amyotrophic lateral sclerosis motor symptoms be explained by prion-like propagation? J Neurol Neurosurg Psychiatry. 2012; 83:739745. [PubMed: 22544947]

49. Kaplan, E.; Goodglass, H.; Weintraub, S., editors. Boston naming test. Lippincott Williams and Wilins; Philadelphia: 2001.

50. Kersaitis C, Halliday GM, Kril JJ. Regional and cellular pathology in frontotemporal dementia: relationship to stage of disease in cases with and without Pick bodies. Acta Neuropathol. 2004; 108:515-523. [PubMed: 15368070]

51. Kiernan MC, Vucic S, Cheah BC, et al. Amyotrophic lateral sclerosis. Lancet. 2011; 377:942-955. [PubMed: 21296405]

52. Kril JJ, Halliday GM. Clinicopathological staging of frontotemporal dementia severity: correlation with regional atrophy. Dement geriatr Cogn Disord. 2004; 17:311-315. [PubMed: 15178943]

53. Kril JJ, Halliday GM. Pathological staging of frontotemporal lobar degeneration. J Mol Neurosci. 2011; 45:379-383. [PubMed: 21552993]

54. Kril JJ, Macdonald V, Patel S, et al. Distribution of brain atrophy in behavioral variant frontotemporal dementia. J Neurol Sci. 2005; 232:83-90. [PubMed: 15850587]

55. Krueger CE, Dean DL, Rosen HJ, et al. Longitudinal rates of lobar atrophy in frontotemporal dementia, semantic dementia, and Alzheimer's disease. Alzheimer Dis Assoc Disord. 2010; 24:4348. [PubMed: 19571735]

56. Kumfor F, Irish M, Hodges JR, et al. The orbitofrontal cortex is involved in emotional enhancement of memory: evidence from the dementias. Brain. 2013; 136:2992-3003. [PubMed: 23838694]

57. Lee EB, Lee VM, Trojanowski JQ. Gains or losses: molecular mechanisms of TDP43-mediated neurodegeneration. Nat rev Neurosci. 2012; 13:38-50. [PubMed: 22127299]

58. Lee EB, Leng LZ, Zhang B, et al. Targeting amyloid-beta peptide (Abeta) oligomers by passive immunization with a conformation-selective monoclonal antibody improves learning and memory in Abeta precursor protein (APP) transgenic mice. J Biol Chem. 2006; 281:4292-4299. [PubMed: 16361260]

59. Lezak, M. Neuropsychological assessment. Oxford University Press; New York: 1983.

60. Li JY, Englund E, Holton JL, et al. Lewy bodies in grafted neurons in subjects with Parkinson's disease suggest host-to-graft disease propagation. Nat Med. 2008; 14:501-503. [PubMed: 18391963]

61. Lillo P, Garcin B, Hornberger M, et al. Neurobehavioral features in frontotemporal dementia with amyotrophic lateral sclerosis. Arch Neurol. 2010; 67:826-830. [PubMed: 20625088]

62. Lillo P, Mioshi E, Burrell JR, et al. Grey and white matter changes across the amyotrophic lateral sclerosis-frontotemporal dementia continuum. PLoS ONE. 2012; 7:e43993. [PubMed: 22952843] 
63. Ling SC, Polymenidou M, Cleveland DW. Converging mechanisms in ALS and FTD: disrupted RNA and protein homeostasis. Neuron. 2013; 79:416-438. [PubMed: 23931993]

64. Liu W, Miller BL, Kramer JH, et al. Behavioral disorders in the frontal and temporal variants of frontotemporal dementia. Neurology. 2004; 62:742-748. [PubMed: 15007124]

65. Lomen-Hoerth C, Anderson T, Miller B. The overlap of amyotrophic lateral sclerosis and frontotemporal dementia. Neurology. 2002; 59:1077-1079. [PubMed: 12370467]

66. Lu MT, Preston JB, Strick PL. Interconnections between the prefrontal cortex and the premotor areas in the frontal lobe. J Comp Neurol. 1994; 341:375-392. [PubMed: 7515081]

67. Luk KC, Kehm VM, Zhang B, et al. Intracerebral inoculation of pathological alpha-synuclein initiates a rapidly progressive neurodegenerative alpha-synucleinopathy in mice. J Exp Med. 2012; 209:975-986. [PubMed: 22508839]

68. Mackenzie IR, Neumann M, Baborie A, et al. A harmonized classification system for FTLD-TDP pathology. Acta Neuropathol. 2011; 122:111-113. [PubMed: 21644037]

69. Mackenzie IR, Neumann M, Bigio EH, et al. Nomenclature for neuropathologic subtypes of frontotemporal lobar degeneration: consensus recommendations. Acta Neuropathol. 2009; 117:1518. [PubMed: 19015862]

70. Mann DM, South PW. The topographic distribution of brain atrophy in frontal lobe dementia. Acta Neuropathol. 1993; 85:334-340. [PubMed: 8460535]

71. Mckhann GM, Albert MS, Grossman M, et al. Clinical and pathological diagnosis of frontotemporal dementia: report of the Work Group on Frontotemporal Dementia and Pick's Disease. Arch Neurol. 2001; 58:1803-1809. [PubMed: 11708987]

72. Mioshi E, Hsieh S, Savage S, et al. Clinical staging and disease progression in frontotemporal dementia. Neurology. 2010; 74:1591-1597. [PubMed: 20479357]

73. Mougenot AL, Nicot S, Bencsik A, et al. Prion-like acceleration of a synucleinopathy in a transgenic mouse model. Neurobiol Aging. 2011; 33:2225-2228. [PubMed: 21813214]

74. Munoz-ruiz MA, Hartikainen P, Koikkalainen J, et al. Structural MRI in frontotemporal dementia: comparisons between hippocampal volumetry, tensor-based morphometry and voxel-based morphometry. PLoS ONE. 2012; 7:e52531. [PubMed: 23285078]

75. Neumann M, Kwong LK, Lee EB, et al. Phosphorylation of S409/410 of TDP-43 is a consistent feature in all sporadic and familial forms of TDP-43 proteinopathies. Acta Neuropathol. 2009; 117:137-149. [PubMed: 19125255]

76. Neumann M, Sampathu DM, Kwong LK, et al. Ubiquitinated TDP-43 in frontotemporal lobar degeneration and amyotrophic lateral sclerosis. Science. 2006; 314:130-133. [PubMed: 17023659]

77. Nishihira Y, Tan CF, Toyoshima Y, et al. Sporadic amyotrophic lateral sclerosis: widespread multisystem degeneration with TDP-43 pathology in a patient after long-term survival on a respirator. Neuropathology. 2009; 29:689-696. [PubMed: 19170893]

78. Nonaka T, Masuda-Suzukake M, Arai T, et al. Prion-like properties of pathological TDP-43 aggregates from diseased brains. Cell Rep. 2013; 4:124-134. [PubMed: 23831027]

79. O'Callaghan C, Bertoux M, Hornberger M. Beyond and below the cortex: the contribution of striatal dysfunction to cognition and behaviour in neurodegeneration. J Neurol Neurosurg Psychiatry. 2013 Epub ahead of print.

80. Ozawa T, Paviour D, Quinn NP, et al. The spectrum of pathological involvement of the striatonigral and olivopontocerebellar systems in multiple system atrophy: clinicopathological correlations. Brain J Neurol. 2004; 127:2657-2671.

81. Perry RJ, Graham A, Williams G, et al. Patterns of frontal lobe atrophy in frontotemporal dementia: a volumetric MRI study. Dement Geriatr Cogn Disord. 2006; 22:278-287. [PubMed: 16914925]

82. Phukan J, Pender NP, Hardiman O. Cognitive impairment in amyotrophic lateral sclerosis. Lancet Neurol. 2007; 6:994-1003. [PubMed: 17945153]

83. Piguet O, Hornberger M, Mioshi E, et al. Behavioural-variant frontotemporal dementia: diagnosis, clinical staging, and management. Lancet Neurol. 2011; 10:162-172. [PubMed: 21147039]

84. Polymenidou M, Cleveland DW. Prion-like spread of protein aggregates in neurodegeneration. J Exp Med. 2012; 209:889-893. [PubMed: 22566400] 
85. Polymenidou M, Cleveland DW. The seeds of neurode-generation: prion-like spreading in ALS. Cell. 2011; 147:498-508. [PubMed: 22036560]

86. Porrino LJ, Crane AM, Goldman-Rakic PS. Direct and indirect pathways from the amygdala to the frontal lobe in rhesus monkeys. J Comp Neurol. 1981; 198:121-136. [PubMed: 6164704]

87. Rabinovici GD, Seeley WW, Kim EJ, et al. Distinct MRI atrophy patterns in autopsy-proven Alzheimer's disease and frontotemporal lobar degeneration. Am J Alzheimers Dis Other Demen. 2007; 22:474-488. [PubMed: 18166607]

88. Rascovsky K, Hodges JR, Knopman D, et al. Sensitivity of revised diagnostic criteria for the behavioural variant of frontotemporal dementia. Brain. 2011; 134:2456-2477. [PubMed: 21810890]

89. Ravits JM, La Spada AR. ALS motor phenotype heterogeneity, focality, and spread: deconstructing motor neuron degeneration. Neurology. 2009; 73:805-811. [PubMed: 19738176]

90. Redgrave P, Rodriguez M, Smith Y, et al. Goal-directed and habitual control in the basal ganglia: implications for Parkinson's disease. Nat rev Neurosci. 2010; 11:760-772. [PubMed: 20944662]

91. Rempel-Clower, Nl; Barbas, H. The laminar pattern of connections between prefrontal and anterior temporal cortices in the rhesus monkey is related to cortical structure and function. Cereb Cortex. 2000; 10:851-865. [PubMed: 10982746]

92. Renton AE, Majounie E, Waite A, et al. A hexanucleo-tide repeat expansion in C9ORF72 is the cause of chromosome 9p21-linked ALS-FTD. Neuron. 2011; 72:257-268. [PubMed: 21944779]

93. Rockland KS, Pandya DN. Laminar origins and terminations of cortical connections of the occipital lobe in the rhesus monkey. Brain Res. 1979; 179:3-20. [PubMed: 116716]

94. Rohrer JD, Geser F, Zhou J, et al. TDP-43 subtypes are associated with distinct atrophy patterns in frontotemporal dementia. Neurology. 2010; 75:2204-2211. [PubMed: 21172843]

95. Rosso SM, Donker Kaat L, Baks T, et al. Frontotemporal dementia in The Netherlands: patient characteristics and prevalence estimates from a population-based study. Brain. 2003; 126:20162022. [PubMed: 12876142]

96. Safar J, Cohen FE, Prusiner SB. Quantitative traits of prion strains are enciphered in the conformation of the prion protein. Archiv Virol. 2000:227-235.

97. Seelaar H, Rohrer JD, Pijnenburg YA, et al. Clinical, genetic and pathological heterogeneity of frontotemporal dementia: a review. J Neurol Neurosurg Psychiatry. 2011; 82:476-486. [PubMed: 20971753]

98. Snowden JS, Rollinson S, Thompson JC, et al. Distinct clinical and pathological characteristics of frontotemporal dementia associated with C9ORF72 mutations. Brain. 2012; 135:693-708. [PubMed: 22300873]

99. Snowden JS, Thompson JC, Stopford CL, et al. The clinical diagnosis of early-onset dementias: diagnostic accuracy and clinicopathological relationships. Brain. 2011; 134:2478-2492. [PubMed: 21840888]

100. Tartaglia MC, Zhang Y, Racine C, et al. Executive dys-function in frontotemporal dementia is related to abnormalities in frontal white matter tracts. J Neurol. 2012; 259:1071-1080. [PubMed: 22037958]

101. The Lund and Manchester Groups. Clinical and neuro-pathological criteria for frontotemporal dementia. J Neurol Neurosurg Psychiatry. 1994; 57:416-418. [PubMed: 8163988]

102. Toledo JB, Van Deerlin VM, Lee EB, et al. A platform for discovery: the University of Pennsylvania integrated neurodegenerative disease biobank. Alzheimers Dement. 2013 doi: 10.1016/j.jalz.2013.06.003.

103. Van De Pol LA, Hensel A, Van Der Flier WM, et al. Hippocampal atrophy on MRI in frontotemporal lobar degeneration and Alzheimer's disease. J Neurol Neurosurg Psychiatry. 2006; 77:439-442. [PubMed: 16306153]

104. Volpicelli-Daley LA, Luk KC, Patel TP, et al. Exogenous alpha-synuclein fibrils induce Lewy body pathology leading to synaptic dysfunction and neuron death. Neuron. 2011; 72:57-71. [PubMed: 21982369]

105. Watts GD, Wymer J, Kovach MJ, et al. Inclusion body myopathy associated with Paget disease of bone and frontotemporal dementia is caused by mutant valosin-containing protein. Nat Genet. 2004; 36:377-381. [PubMed: 15034582] 
106. Whitwell JL, Jack CR Jr, Parisi Je, et al. Imaging signatures of molecular pathology in behavioral variant frontotemporal dementia. J Mol Neurosci. 2011; 45:372-378. [PubMed: 21556732]

107. Whitwell JL, Sampson EL, Watt HC, et al. A volumetric magnetic resonance imaging study of the amygdala in fronto-temporal lobar degeneration and Alzheimer's disease. Dement Geriatr Cogn Disord. 2005; 20:238-244. [PubMed: 16088140]

108. Wu CW, Bichot NP, Kaas JH. Converging evidence from microstimulation, architecture, and connections for multiple motor areas in the frontal and cingulate cortex of prosimian primates. $\mathrm{J}$ Comp Neurol. 2000; 423:140-177. [PubMed: 10861543]

109. Xie SX, Baek Y, Grossman M, et al. Building an integrated neurodegenerative disease database at an academic health center. Alzheimers Dement. 2011; 7:e84-e93. [PubMed: 21784346]

110. Yakovlev PI. Motility, behavior and the brain; stereodynamic organization and neural coordinates of behavior. J Nerv Ment Dis. 1948; 107:313-335. [PubMed: 18913439]

111. Yu CE, Bird TD, Bekris LM, et al. The spectrum of mutations in progranulin: a collaborative study screening 545 cases of neurodegeneration. Arch Neurol. 2010; 67:161-170. [PubMed: 20142524] 


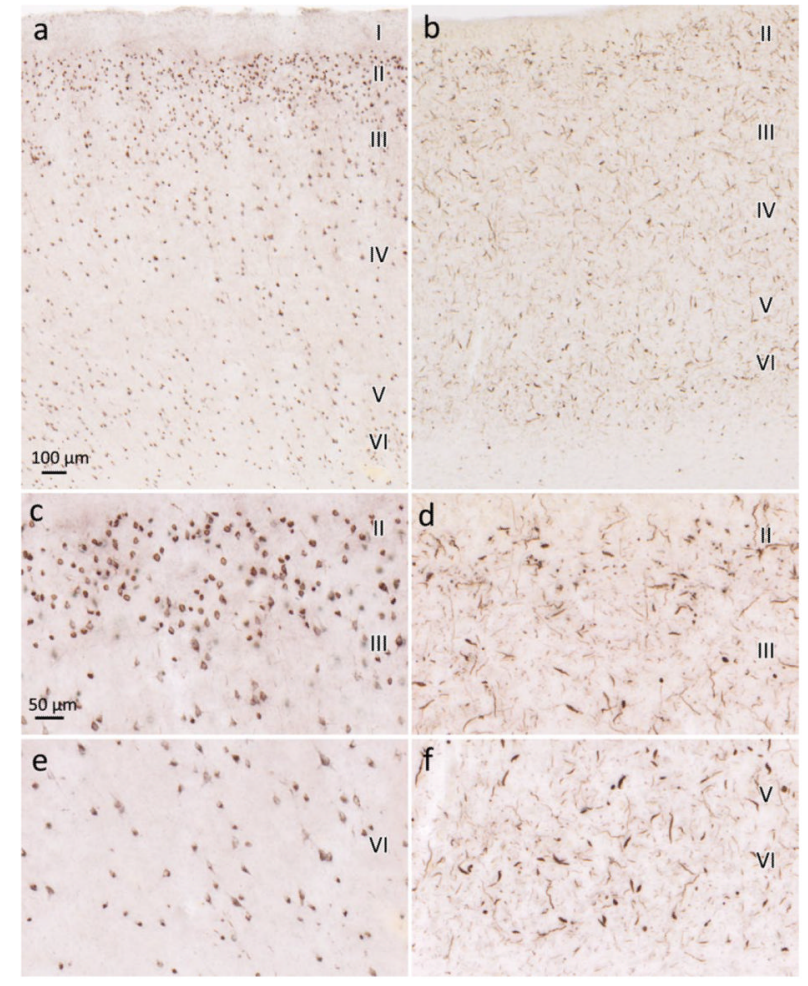

Fig. 1.

Subtypes of cortical pTDP-43 pathology in FTLD. pTDP-43 IHC shows pTDP-43 pathology in the anterior cingulate gyrus thatp resented either with predominantly perikaryal pTDP-43 inclusions (cytoplasmic type in $\mathbf{a}, \mathbf{c}, \mathbf{e}$ ) or with long and extensive aggregates in dendrites (neuritic type in $\mathbf{b}, \mathbf{d}, \mathbf{f}$ ). Both the neuritic type and the cytoplasmict ype showed prominent involvement of projection neurons of cortical layers $\boldsymbol{I I}$ and $\boldsymbol{I I I}(\mathbf{c}, \mathbf{d})$, although the cytoplasmic type more often showed involvement of Betz pyramidal cells of layer $\mathrm{Vb}$ in the primary motor cortex than the neuritic type. Scale in $\mathbf{a}$ applies to $\mathbf{b}$; scale bar in $\mathbf{c}$ is also valid for $\mathbf{d}-\mathbf{f}$ 


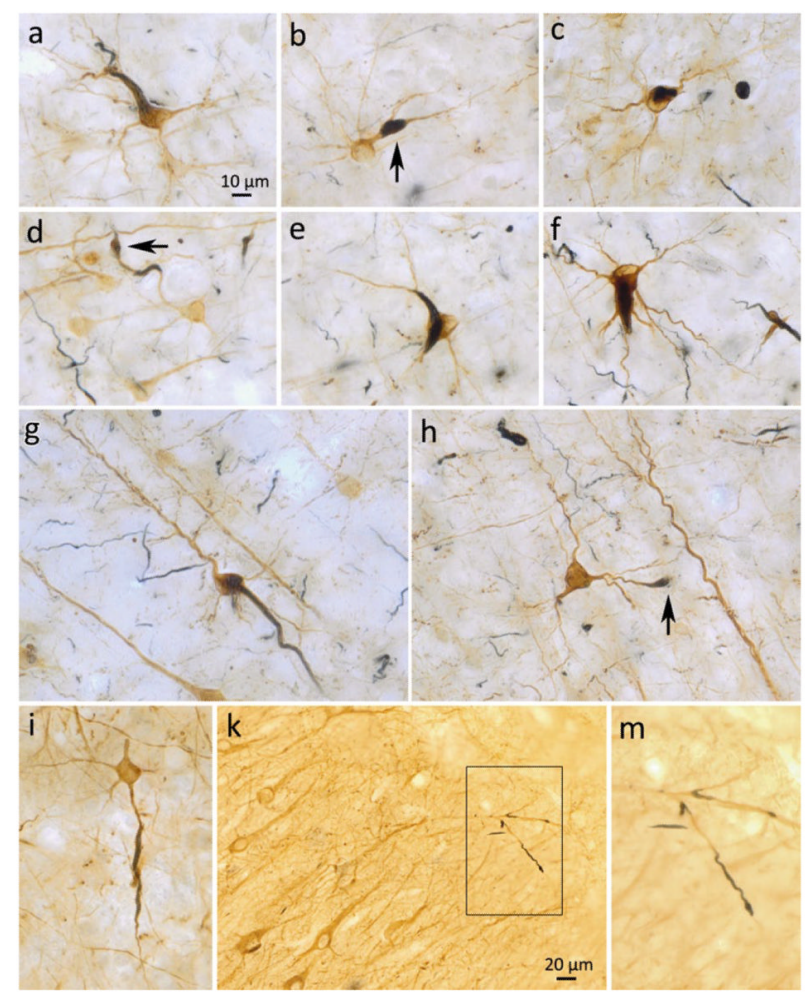

Fig. 2.

pTDP-43 pathology in dendrites of neuritic type FTLD-TDP. Double-labeling IHC of the middle frontal gyrus using pTDP-43 antibody (dark blue) and an anti-neurofilament marker (SMI-311, a-i) or the anti-neurofilament antibody NFH 200 (k, m) (brown). a pTDP-43 aggregates in the neuronal perikaryon and proximal parts of a basal dendrite. $\mathbf{b}$ pTDP-43 aggregates at the ramification of basal dendrite showing a focal (arrow) bulb-like dendritic swelling. c Occasionally, neuritic type cases also showed intra-perikaryonal pTDP-43 aggregates. d Elongated pTDP-43 lesion in a basal dendrite that shows curvatures and a focal (arrow) dendritic swelling. e Intraneuronal pTDP-43 aggregate encompassing part of the perikaryon as well as the proximal portions of two dendrites. $\mathbf{f}, \mathbf{g}$ pTDP-43 aggregates in the perikaryon and proximal portion of an apical dendrite. $\mathbf{h}$ Bulbous swelling (arrow) of a basal dendrite caused by pTDP-43 aggregates. i-m pTDP-43 aggregates in apical dendrites and their ramifications $(\mathbf{k}, \mathbf{m})$; framed area in $\mathbf{k}$ is shown at higher resolution in $\mathbf{m}$. Scale bar in a also applies to $\mathbf{b}-\mathbf{i}$ and $\mathbf{m}$ 

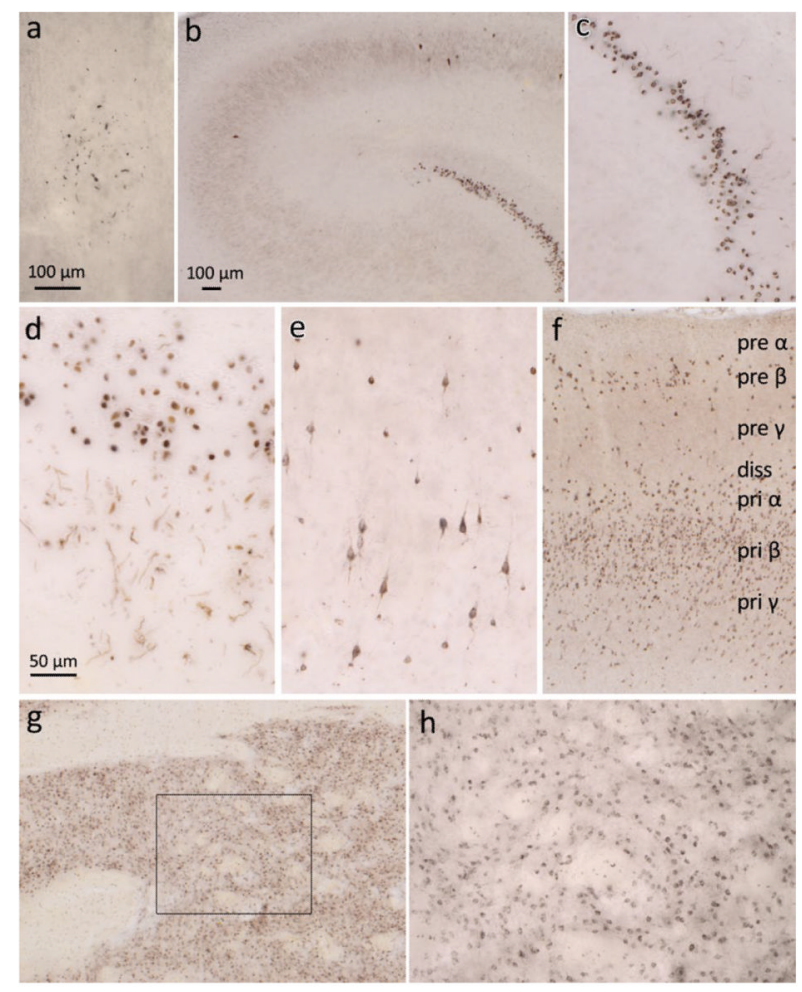

Fig. 3.

pTDP-43 pathology in the olfactory, mesiotemporal regions, and striatal regions of FTLDTDP. a the anterior olfactory nucleus contains pTDP-43-immunoreactive inclusions. b pTDP-43 IHC shows extensive pathology in granular cells of the hippocampal fasciad entata and in dendrites of molecular layer originating from granularc ells. $\mathbf{c}$ dare higher resolution excerpts from b. e pyramidal neurons of the Ammon's horn (CA1) containing pTDP-43 inclusions. $\mathbf{f}$ pTDP-43 pathology in the entorhinal cortex affecting mainly layers pre- $\beta$ as well as pri- $\alpha$ and pri- $\beta$. $\mathbf{g}, \mathbf{h}$ Striatal pTDP-43 pathology in the accumbens nucleus affecting mainly medium-sized projection neurons; $h$ is a higher resolution excerpt from the framed area in $\mathbf{g}$. Scale bar in $\mathbf{a}$ is also valid for $\mathbf{c}, \mathbf{e}$, and $\mathbf{h}$; scale bar in $\mathbf{b}$ applies to $\mathbf{f}$ and $\mathbf{g}$ 


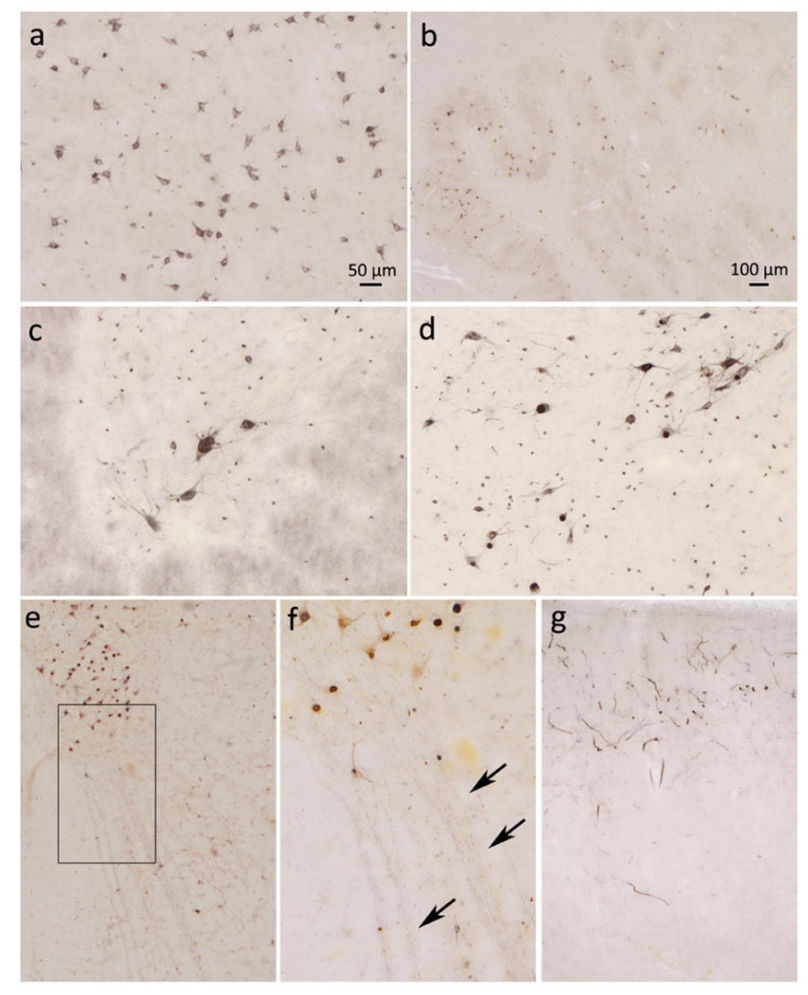

Fig. 4.

Thalamic, bulbar, spinal cord, and occipital pTDP-43 pathology in FTLD-TDP. a pTDP-43 IHC pTDP-43 pathology in cortically projecting thalamic neurons. b pTDP-43 aggregates in cells of the inferior olive. $\mathbf{c}$ Cervical spinal cord anterior horn showing pTDP-43 pathology in a-motoneurons as well as dot-like or circumferential inclusions in oligodendroglia. $\mathbf{d}$ pTDP-43 aggregates in hypoglossal nucleus (XII) neurons of the medulla oblongata. e, $\mathbf{f}$ Hypoglossal nucleus and intramedullary axons of this nucleus with pTDP-43 aggregates. Framed area in $\mathbf{e}$ is shown at higher resolution in $\mathbf{f} . \mathbf{f}$ Arrows indicate the bundles of intramedullary axons. $\mathbf{g}$ Visual cortex pTDP-43 pathology (peristriate region, Brodmann area 17,18 ) as observed in pattern IV cases. Scale bar in $\mathbf{a}$ is also valid in $\mathbf{c ,} \mathbf{d}, \mathbf{f}$, and $\mathbf{g}$; scale bar in $\mathbf{b}$ also applies to $\mathbf{e}$ 
a

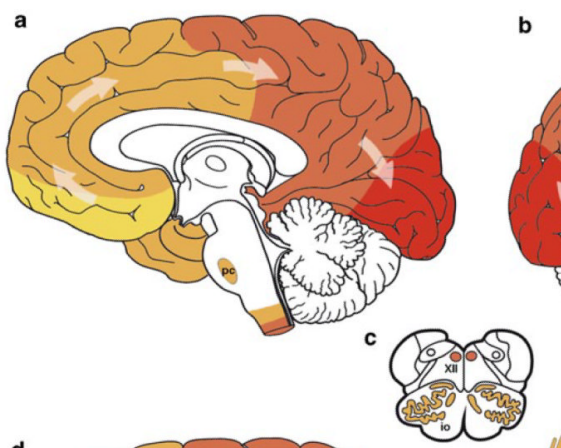

d

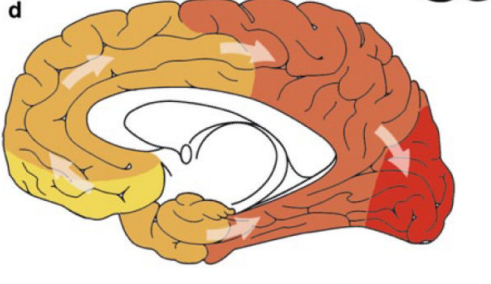

pattern I

pattern II

pattern III
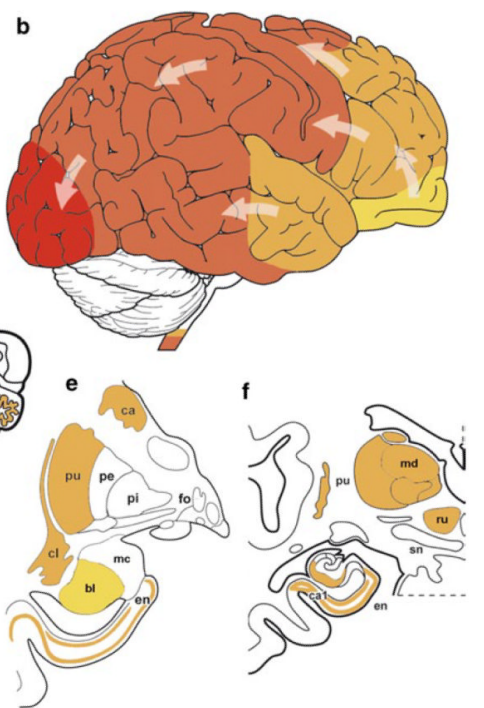

pattern IV

Fig. 5.

Summary diagram of four regional distribution patterns of pathology in bvFTLD illustrating the hypothesis that pTDP-43 pathology may spread sequentially, possibly propagating (white arrows) along axonal pathways. Preferentially vulnerable cortical and subcortical sites are involved. Pattern I cases with the lowest burden of pTDP pathology display the presence of pTDP-43 immunoreactive inclusions in projection neurons and oligodendrocytes of basal and anterior portions of the prefrontal neocortex (orbital gyri, gyrus rectus, inferior frontal gyrus). Pattern II cases show the same lesion distribution as pattern I, but with pTDP pathology also in prefrontal neocortical areas located further caudal to the frontal pole, including the middle frontal gyrus, insular cortex, and anterior cingulate gyrus, and also in anteromedial areas (transentorhinal region, adjoining allocortical entorhinal region and hippocampal formation), the superior and middle temporal gyri, striatum (accumbens nucleus, putamen, claustrum), parvocellular portion of the red nucleus, medial and lateral portions of the thalamus, and in precerebellar nuclei of the pons and medulla oblongata. Cases with additional pathology beyond that seen in the first two patterns are characterized by the presence of pTDP-43 immunoreactive inclusions in the agranular motor neocortex (Brodmann fields 4 and 6), in parietal neocortical areas (sensory cortex, angular gyrus), in bulbar somatomotor neurons, and in a-motoneruons of the cervical spinal cord anterior horns which is pattern III. Cases with the highest burden of pathology (pattern IV) show the presence of pTDP-43 lesions in the occipital neocortex (visual cortex, Brodmann 17, 18) in addition to involvement of all of the areas involved in patterns I-III. a $p c$ precerebellar nuclei; $\mathbf{c} X I I$ hypoglossal nucleus, io inferior olive; $\mathbf{e} c a$ caudate nucleus, $p u$ putamen, $p i$ internal pallidum, $p e$ external pallidum, $f o$ fornix, $c l$ claustrum, $m c$ mediocentral subnuclei of the $b l$ basolateral subnuclei of amygdala, en entorhinal cortex (en); $\mathbf{f} p u$ putamen, $m d$ mediodorsal thalamus, $r n$ red nucleus, $s n$ substantia nigra (sn), cal Ammon's horn region 1, en entorhinal cortex 


\section{Table 1}

Demographic data, neuropsychological testing data, and genetic status of FTLD-TDP cases $(n=39)$ included in this study

\begin{tabular}{ll}
\hline & bvFTD \\
\hline$N$ (female/male) & $39(20 / 19)$ \\
Age at onset (years) & $58(51.8-67)$ \\
Disease duration (years) & $7(4.8-11)$ \\
MMSE & $8.5(5-19)$ \\
FAS & $8(2.3-11)$ \\
BNT & $18(11-21.5)$ \\
C9orf72 & $30.8 \%$ \\
GRN & $15.4 \%$ \\
VCP & $0 \%$ \\
\hline
\end{tabular}

Data are shown as median and interquartile range in brackets

FTLD frontotemporal lobar degeneration, $b v F T D$ behavioral variant frontotemporal dementia, MMSE Mini-Mental State Examination, FAS test of letter-guided verbal fluency, BNT Boston Naming Test, $C 9$ orf 72 frequency of the C9orf72 hexanucleotide repeat expansion within a clinical subgroup, $G R N$ frequencyof a progranulin gene mutation within a clinical subgroup, $V C P$ frequency of a valosin-containing protein mutation within a clinical subgroup 


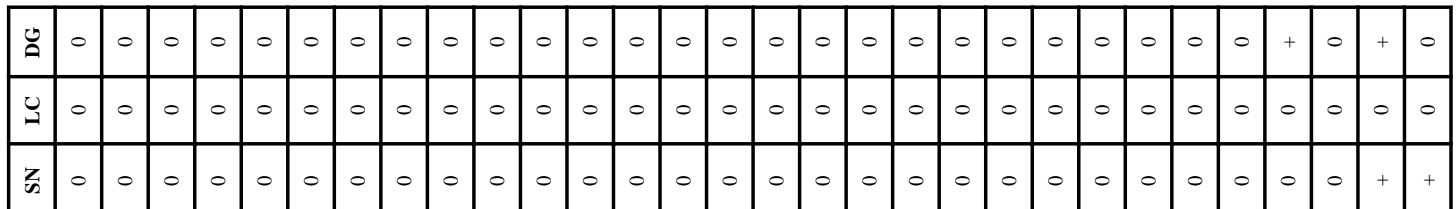

\begin{tabular}{lllllllllllllllllllllllllllllllllllll}
$\frac{n}{5}$ & 0 & 0 & 0 & 0 & 0 & 0 & 0 & 0 & 0 & 0 & 0 & 0 & 0 & 0 & 0 & 0 & 0 & 0 & 0 & 0 & 0 & 0 & 0 & 0 & 0 & 0 & + & + & + & + & + \\
\hline
\end{tabular}

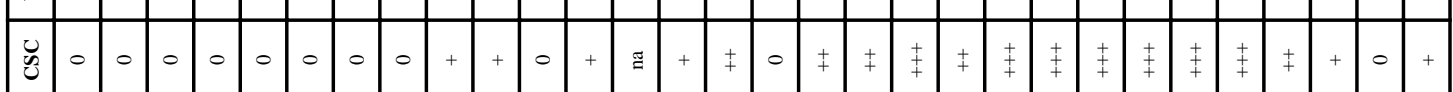

\section{$\bar{\Sigma}$}

帘

峞

:

E

za

z

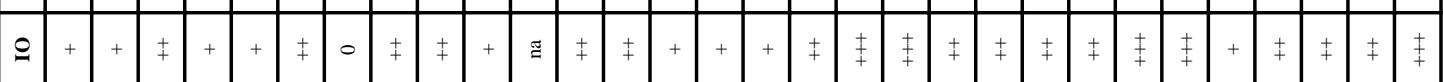

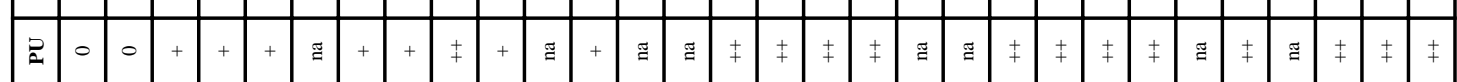

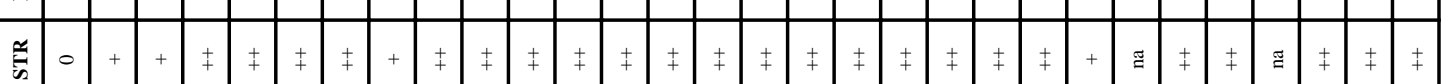

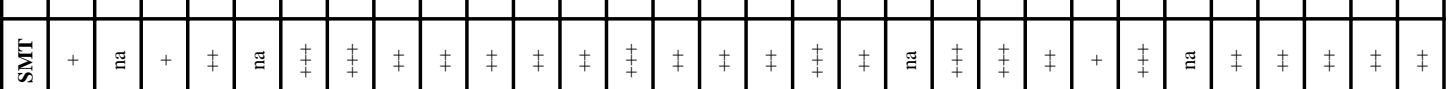

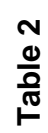

异

\begin{tabular}{|c|c|c|c|c|c|c|c|c|c|c|c|c|c|c|c|c|c|c|c|c|c|c|c|c|c|c|}
\hline$\overline{\mathbf{E}} \mid$ & & + & & & & & & & & & & & & & & & 丰 & & & & & & & 去 & & \\
\hline$\overline{\mathbf{z}}$ & + & + & 辛 & & & 寿 & & & & & & & & & & 寿 & $\mid \neq$ & & & & & & & 丰 & & \\
\hline$\frac{1}{2}$ & + & $\ddagger$ & $\ddagger$ & & $\ddagger$ & gू & & & + & & ${ }^{+}$ & & & & & & & & & $\ddagger$ & & $\mp$ & & $\ddagger$ & & \\
\hline$\frac{\pi}{2}$ & & $\mp$ & & & & 丰 & & & 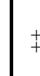 & & $\ddagger$ & 寿 & & & & & + & & & & & & & $\neq$ & & \\
\hline \& & & $\neq$ & $\ddagger$ & $\mid \neq$ & 丰 & 丰 & † & & 韦 & $\ddagger$ & 耒 & 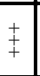 & & & & $1 \neq$ & 䏝 & 辛 & 丰 & & & 䒠 & & 丰 & & \\
\hline 气ิ & $\bar{I}=$ & : & $=$ & $=$ & $=$ & $=$ & $=$ & & $\equiv$ & & $\equiv$ & $\equiv$ & - & & & $\equiv$ & $\equiv$ & $\Xi$ & $\equiv$ & $\equiv$ & & & $\geq$ & $\geq$ & & \\
\hline$\frac{\hat{z}}{\frac{1}{2}}$ & & $=$ & $\equiv$ & & $=$ & & & 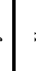 & $=$ & $\Xi$ & $=$ & $=$ & $=1$ & & & 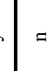 & $\Rightarrow$ & $\therefore$ & & $=$ & & & & 1 & & \\
\hline$\hat{\mathrm{z}}$ & $\therefore$. & 。 & $\circ$ & m & 0 & $\circ$ & $=$ & . & Io & 0 & $\circ$ & 0 & 0 & & 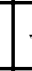 & ${ }^{\circ}$ & 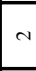 & - & $\therefore$ & 0 & & $\circ$ & $\infty$ & 0 & & \\
\hline 文 & & - & - & $\equiv$ & - & $\circ$ & b & 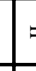 & 10 & & o & - & 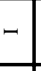 & 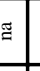 & 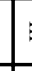 & 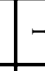 & 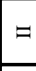 & - & 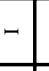 & - & & $=$ & $\equiv$ & $\Rightarrow$ & & $\equiv$ \\
\hline$\frac{2}{\frac{s}{4}}$ & & $\varangle$ & $\infty$ & $\varangle$ & $<$ & $<1$ & & & 8 & œ & $\varangle$ & $\varangle$ & $m$ & & & $\infty$ & $\varangle$ & $\infty$ & $\infty$ & $<$ & & & & 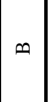 & & \\
\hline & & & & & 館 & 害 & & & E & & & $\exists$ & & & & & 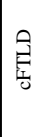 & & 욜 & & & & & 㝕 & & \\
\hline & & & 8 & & 棁 & & & & 䂞 & & 즘 & $\bar{\Sigma}$ & 8 & & & & & 8 & 8 & 彦 & & & 8 & i & & \\
\hline \begin{tabular}{|l|} 
\\
\end{tabular} & & 5 的 & : & & in & 品 & & & F & F & & : & 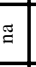 & & & 10 & $\stackrel{2}{2}$ & $\bar{g}$ & $\therefore$ & $\frac{10}{4}$ & $7^{\circ}$ & 8 & $\bar{n}$ & 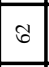 & & \\
\hline 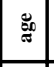 & & 0 & $\therefore$ & 8 & s & $\therefore$ & & & I & $\kappa$ & $\sigma$ & 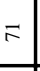 & o & & & $\tilde{\sigma}$ & $\stackrel{\infty}{\infty}$ & $\therefore$ & in & & $f=$ & & : & $F$ & & \\
\hline$\Xi$ & & - & - & $\Xi$ & - & $\Xi$ & & & 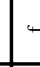 & 4 & $\Xi$ & $\Xi$ & $\Xi$ & & & & $\Xi$ & $\Xi$ & & - & $\equiv$ & & - & - & & \\
\hline & & & + & & & & & & & & & & & & & & & & $\approx 1$ & & & & ¿ & $\infty$ & & \\
\hline
\end{tabular}




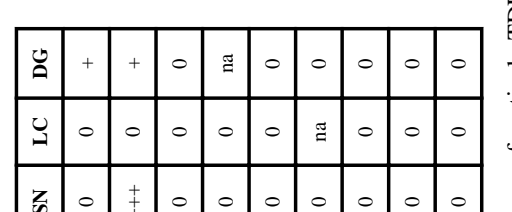

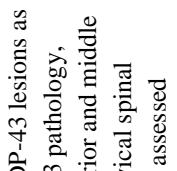

年

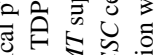

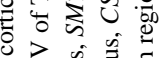

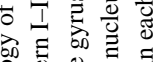

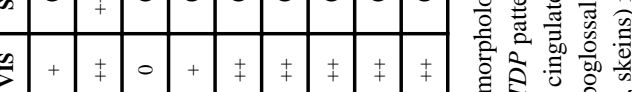

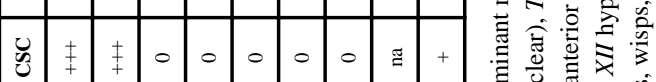

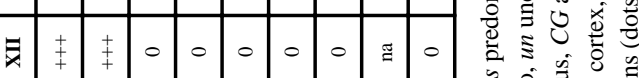

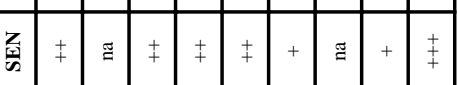

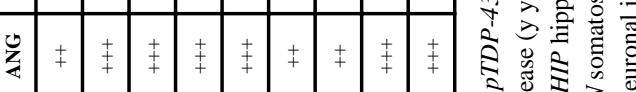

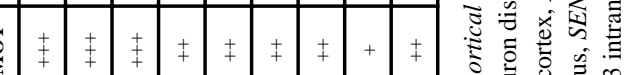

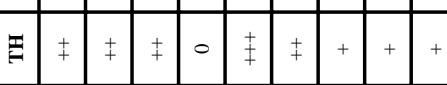

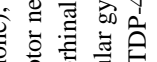
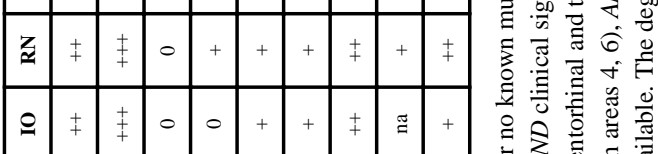

零

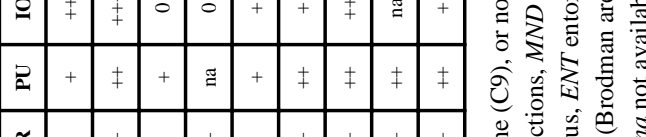

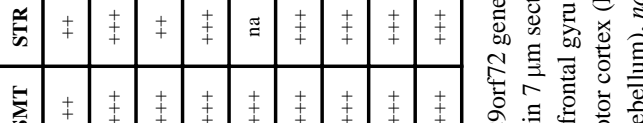

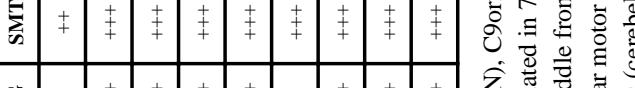

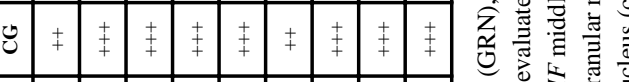

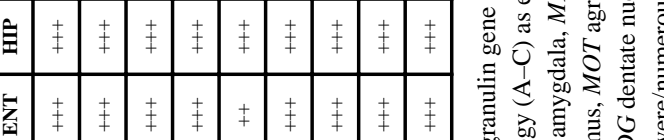

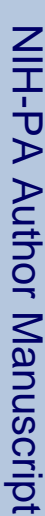

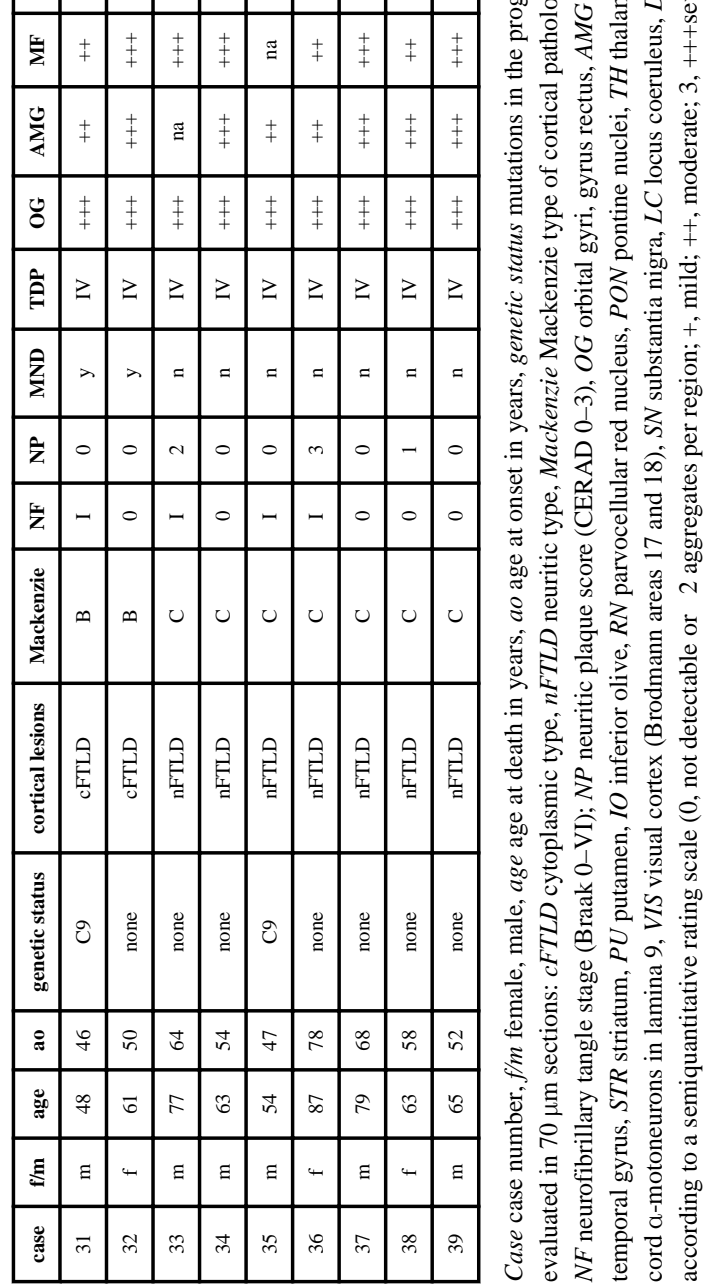

